This item was submitted to Loughborough's Research Repository by the author.

Items in Figshare are protected by copyright, with all rights reserved, unless otherwise indicated.

\title{
Interleukin-10 responses from acute exercise in healthy subjects: A systematic review
}

PLEASE CITE THE PUBLISHED VERSION

https://doi.org/10.1002/jcp.27920

PUBLISHER

(c) Wiley

VERSION

AM (Accepted Manuscript)

\section{PUBLISHER STATEMENT}

This is the peer reviewed version of the following article: CABRAL-SANTOS, C. ... et al., 2018. Interleukin-10 responses from acute exercise in healthy subjects: A systematic review. Journal of Cellular Physiology, 234 (7), pp.9956-9965, which has been published in final form at https://doi.org/10.1002/jcp.27920. This article may be used for non-commercial purposes in accordance with Wiley Terms and Conditions for Use of SelfArchived Versions.

\section{LICENCE}

CC BY-NC-ND 4.0

\section{REPOSITORY RECORD}

Cabral-Santos, Carolina, Edson Alves de Lima Junior, Isabela Maia da Cruz Fernandes, Rafael Zambelli Pinto, Jose Cesar Rosa-Neto, Nicolette Bishop, and Fabio S. Lira. 2019. "Interleukin-10 Responses from Acute Exercise in Healthy Subjects: A Systematic Review". figshare. https://hdl.handle.net/2134/37036. 


\section{Interleukin-10 responses from acute exercise in healthy subjects: a systematic review}

\section{Short running title: IL-10 and acute exercise}

Carolina Cabral-Santos ${ }^{1}$, Edson Alves de Lima Junior ${ }^{3}$, Isabela Maia da Cruz Fernandes ${ }^{1}$, Rafael Zambelli de Almeida Pinto ${ }^{2}$, José César Rosa-Neto ${ }^{3}$, Nicolette Claire Bishop ${ }^{4}$, Fábio Santos Lira ${ }^{1}$

${ }^{1}$ Exercise and Immunometabolism Research Group, Post-Graduation Program in Movement Sciences, Department of Physical Education, Universidade Estadual Paulista (UNESP) - Rua Roberto Simonsen, 305, CEP 19060-900, Presidente Prudente, São Paulo, Brazil.

${ }^{2}$ Department of Physical Therapy, Universidade Federal de Minas Gerais (UFMG), Belo Horizonte, Brazil.

${ }^{3}$ Immunometabolism Research Group, Department of Cell and Developmental Biology, University of São Paulo, São Paulo, Brazil - Av Prof Lineu Prestes, 1524, CEP 05508-900, Butantã, São Paulo, Brazil.

${ }^{4}$ School of Sport, Exercise, and Health Sciences and National Centre for Sport and Exercise Medicine, Loughborough University, Loughborough, LE11 3TU, United Kingdom

Corresponding author:

Fábio Santos Lira

Exercise and Immunometabolism Research Group

Department of Physical Education - University of the State of São Paulo (UNESP)

Rua Roberto Simonsen, 305, CEP 19060-900, Presidente Prudente, SP, Brazil.

Phone: 5518 3229-5826 / Fax: 5518 3229-5710

E-mail address: fabio.lira@,unesp.br

\section{Acknowledgements}

This study was funded by FAPESP (grant number 2015/17068-2, 2016/16712-8, 2015/26148-0 and 2016/14098). This study was financed in part by the Coordenação de Aperfeiçoamento de Pessoal de Nível Superior Brasil (CAPES) - Finance Code 001. 


\begin{abstract}
Purpose: Interleukin 10 (IL-10) is a cytokine that plays a critical role with potent anti-inflammatory properties when produced during exercise, limiting host immune response to pathogens and preventing tissue damage. The purpose of this systematic review was to assess the response of IL-10 after acute exercise session in healthy adults. Methods: Databases of Ovid Medline (1978-2016), CINAHL (1998-2016), EMBASE (2003-2016), SportDiscus (1990-2016), and Web of Science library (1990-2016) were carefully screened. Clinical trials comparing exercise types in healthy individuals were included for pooled analysis. The trials of exercise were methodologically appraised by PEDro Scale. Results: 12 randomized controlled and crossover trials containing 176 individuals were identified for inclusion. The Kruskal-Wallis test showed no significant differences between type of exercise and the corresponding values in IL-10 $[\mathrm{X} 2(4)=2.878 ; \mathrm{p}=0.449]$. The duration of exercise was significantly correlated with increase in IL-10 changes [Pearson's $\mathrm{r}=1.00,95 \% \mathrm{CI}$ : 0.015 to $0.042, \mathrm{p}<0.0001$ ] indicating that $48 \%$ of the variation in IL-10 levels can be explained by the duration of the exercise performed. In addition, despite a linear increase, we did not find significant correlation with the intensity of exercise and IL-10 changes [Pearson's r $=0.218,95 \% \mathrm{CI}$ : -0.554 to $0.042, \mathrm{p}<0.035$ ]. Conclusion: Overall, the duration of the exercise is the single most important factor determining the magnitude of the exercise-induced increase of plasma IL-10.
\end{abstract}

Key-words: Interleukin 10, inflammation, anti-inflammatory response, acute exercise. 


\section{Introduction}

In healthy individuals, acute and chronic exercise is known to alter circulating concentrations of inflammatory markers, reduce incidence of infection and promote a lower risk of chronic disease associated with morbidity (Pedersen and Saltin, 2015). Physical exercise induces strong metabolic and immunological responses - seen by release of some cytokines such as serum and skeletal muscle Interleukin 6 (IL-6), Interleukin 10 (IL10), and Tumor Necrosis Factor-alpha (TNF- $\alpha$ ) - that act on inflammatory responses altering the pro/antiinflammatory balance with crucial role in tissue repair and energy metabolism (Fischer, 2006; Pedersen and Febbraio, 2009). However, these responses are dependent on duration, intensity and therefore the volume of exercise performed in a session (Lira et al. 2012).

IL-10, a small soluble protein that mediates communication between immune and non-immune cells, was first described by Fiorentino et al (1989) and termed cytokine synthesis inhibitory factor (CSIF). It is expressed and endogenously released by cells of immune system including macrophages, dendritic cells, natural killer cells, eosinophils and neutrophils, B cells, several T-cell subpopulations including Th1, Th2 and T-regulatory cells (Kwilasz et al. 2015). IL-10 acts as an immune mediator and inhibits the production of interferon gamma (IFN- $\gamma$ ) and the synthesis of cytokines by T-helper 1 cells (Th1), monocyte, and macrophage (Steensberg et al. 2003; Fiorentino et al. 1989).

The principal role of this interleukin is to prevent the exacerbation of the pro-inflammatory response, blocking a possible persistent inflammatory state (Pedersen and Febbraio, 2009). Classically, IL-10 acts to inhibit cytokine synthesis mainly at the level of cytokine gene transcription (Wang et al. 1994) and has strong down regulatory effects on secretion of pro-inflammatory cytokines such as IL-1, IL-1 $\beta$, TNF- $\alpha$ (Waal Malefyt et al. 1991) which are all involved in chronic inflammatory processes and acute exercise. Furthermore, IL-10 enhances the release of anti-inflammatory mediators such as IL-1 receptor antagonist (IL-1 ra), an antagonistic cytokine that competes with IL-1 for receptor binding without inducing signal transduction, and also soluble TNF receptors (sTNFRs) from innate immune cells (Kwilasz et al. 2015). Dysregulation of IL-10 is associated with impaired immune responses to infection, chronic inflammation and increase risk for development of many autoimmune diseases (Iyer and Cheng, 2012).

Recently, a study by Wadley et al (2016) suggests that the magnitude of increase in concentration of circulating cytokines in response to exercise is dependent on exercise volume. The effects of physical exercise on IL-10 levels have been used to recommend exercise as an anti-inflammatory therapy. Studies have demonstrated that in healthy subjects acute moderate running at $70 \%$ of speed associated with maximum rate of oxygen consumption $\left(\mathrm{sVO}_{2} \mathrm{max}\right)$ and high intensity exercise at $100 \% \mathrm{sVO}_{2}$ max resulted in a significantly increase in IL10 during and after exercise until $60 \mathrm{~min}$ (Cabral-Santos et al. 2015) leading to an temporary increase in the circulating anti-inflammatory status. Rosa-Neto et al (2009) observed that exhaustive exercise exerted an antiinflammatory effect observed by most prominent changes in IL-10 and the IL-10/TNF- $\alpha$ ratio in skeletal muscle (especially in type 2 fibers), where the opposite response was observed in white adipose tissue with proinflammatory effect (increase in the protein expression of IL-6 and TNF- $\alpha$ ) possibly contributing to increased lipolysis to provide energy for the exercising muscle.

Given these documented differences in the effects of acute exercise on IL-10 responses according to intensity and duration (and therefore as a result, volume) it is important to consider these factors when investigating immune responsiveness to exercise performed. Our aim was to assess the effect of acute exercise on 
IL-10 response in healthy subjects. Understanding what are the effect of exercise session and the magnitude required in order to increase IL-10 following different types of exercise program is necessary to provide an evidence-based approach to exercise prescription for individuals.

\section{Methods}

Systematic review registration

The protocol was registered with the International Prospective Register of Systematic Reviews on Nov 15, 2016 at: http://www.crd.york.ac.uk/PROSPERO. FDA device/drug status: Not applicable.

\section{Electronic searches}

A computerized systematic search was first performed in Ovid MEDLINE and subsequently adapted to CINAHL, EMBASE, Web of Science and SPORTDiscus databases. The search was conducted from 1978 to January 2017. The search strategy consisted of a combination of database-specific combinations of key terms (text words contained in the title and abstract, and of the index terms used to describe articles) and Boolean operators ("AND", "OR', "NOT"). The detailed search strategy was performed with the following descriptors: “inflamm $\$$ or biomarkers or immune $\$$ or inflammatory mediators or inflammatory biomarkers or serum mediators or interleukin-10 or IL-10, health\$ or healthy individuals, physical exercise, physical training, continuous exercise, high intensity interval exercise, high intensity interval training, sprint interval training, sprint interval exercise, resistance exercise, endurance exercise, aerobic exercise, strength exercise" and all combined (Appendix 1). The search terms were modified according to the specific vocabulary map of each database.

\section{Study Selection}

Citations were assessed based on all fields. Study selection, full publications of potentially relevant studies, critical quality appraisal and data extraction were conducted independently by two reviewers using the pre-defined eligibility criteria. Disagreements were resolved via discussion, with a third party if necessary, until a consensus was reached.

Additionally, the reference lists of all identified reviews were used Endnote (version X7.3.1, Thomson Reuters, Philadelphia, PA, USA) to create a bibliographic database to manage the search results. Titles and abstract were retrieved and screened by two independent reviewers (CCS, IMCF, EAL).

\section{Inclusion criteria of studies}

Study design: randomized and non-randomized controlled and uncontrolled interventions.

- Acute Exercise and Interleukin-10;

- Participants: healthy individuals without any age restriction;

- Interventions: acute aerobic and/or resistance exercise and/or endurance and/or high intensity interval exercise;

- Outcome measures: all parameters of immune function (e.g. cytokines, mediating proteins, cell counts and functions);

$\cdot$ IMC $\leq 24.9$.

Articles were excluded if they were case reports, if they were written in a language other than English, - 
did not provide enough information, - articles without exercise protocols, articles that used animal models, sample related to a disease, or duplicate publications, and studies without full text manuscript available (e.g. abstracts, conference proceedings, presentations).

\section{Quality assessment}

Methodologic quality was evaluated using the Physiotherapy Evidence based Database criteria - PEDro scale (Moseley et al. 2002). This scale is scored the methodological quality of clinical trials in relation to their internal validity according to 11 items; that indicates the quality in scores range from: high quality (9-10), good $(6-8)$, moderate $(4-5)$, or poor $(<4)$. In case of conflicting evaluations, disagreements were resolved through discussion between the authors.

\section{Statistical analyses}

Descriptive data are shown as means and standard deviation (Table 1). Kruskal-Wallis Analysis of variance was used to compare the delta changes from IL-10 $(\Delta)$ were as the ratio between the pre-exercise values in relation to post-exercise values $[\Delta=$ post-exercise - pre-exercise values] in the type of exercise. All pairwise comparisons were performed using the Dunns method.

Correlations between the exercise duration, intensity and changes in plasma IL-10 were evaluated using Pearson correlation coefficients (r). Statistical significance was set at 5\% for all analysis and the calculations were conducted using GraphPad Prism 7.

\section{Results}

Study selection

The initial electronic database search identified 3.147 articles, of which 2.825 were screened (after removal of duplicates - 322). In total, 2.738 publications were excluded on the basis of title and abstract. On application of the review inclusion criteria to the 57 studies of potential relevance full-text papers, a further 45 papers were excluded and the most consistently reasons for exclusion were do not evaluated IL-10 values in the timepoint pre- and immediately post-exercise or, not collected and evaluated IL-10 from blood samples. The least consistently reason were because exercise protocol utilized was chronic, exercise realized in a climatic chamber, or other placebo supplementation procedures. Therefore, 12 publications met the inclusion criteria and were included in the systematic review. Figure 1 shows a flowchart of the articles search.

\section{**Insert Figure 1 near here**}

\section{Description of studies}

To investigate the effects in IL-10 behavior after acute exercise, the results were grouped according to type of exercise. Four articles studied the effects of exercise and IL-10 on strength (Agostinete et al. 2016; GerosaNeto et al. 2016; Peake et al. 2006; Rossi et al. 2016), three on continuous running versus intermittent running performed on treadmill (Cabral-Santos et al. 2015; Dorneles et al. 2016; Ghafourian et al. 2016), one on cycling ergometer (Cullen et al. 2016;) and four on strenuous exercise - lasting at least 3 hours of outdoor long distance race (which the runners were allowed to choose their own speed) associated or not to other type of exercise 
(Comassi et al. 2015; Kaoru et al. 2013; Krzemiński et al. 2016; Nickel et al. 2011).

\author{
**Insert Table 1 here**
}

\title{
Effects of interventions
}

The Kruskal-Wallis test showed no significant differences between grouped type of exercise and the corresponding values in $\Delta \mathrm{IL}-10\left[\mathrm{X}^{2}(4)=2.878 ; p=0.449\right]$, as shown in Figure 2.10 of the 12 eligible articles showed an increase in IL-10 levels, 2 did not report statistically significant differences after acute exercise.

\footnotetext{
**Insert Figure 2 near here**
}

The duration of exercise was significantly correlated with increase in IL-10 changes (Pearson's r $=1.00$, $95 \%$ CI: 0.0159 to $0.0425, p<0.0001$, Figure 3 ) indicating that $48 \%$ of the variation in IL- 10 levels can be explained by the duration of exercise. However, despite a linear increase there was no correlation of intensity and increase in IL-10 levels (Pearson's r $=0.212,95 \% \mathrm{CI}$ : -0.554 to $0.927, p<0.035$ ).

\author{
**Insert Figure 3 and 4 near here**
}

\section{Discussion}

This study investigated the influence of type and intensity of acute exercise on to better understanding of the amount of exercise is required to increase anti-inflammatory response in health individuals. The physical exercise represent an acceptable model to induce an inflammatory response, commonly seen by increase the mobilization and activation of granulocytes, lymphocytes, and monocytes, as well release of inflammatory factors and soluble mediator (Shek and Shephard, 1998). However, regular physical exercise seems to play an important role in health benefits by increasing IL-10 levels which appears to be a pivotal function for reducing the ability of macrophages to respond to IFN- $\gamma$ and their microbicidal activity, and the negative feedback response to the production of pro-inflammatory cytokines, such as TNF- $\alpha$, IL-1 $\beta$, IL-6, IL-8, IL-12 (Pedersen and Febbraio, 2009; Oswald et ail. 1992). The plasma IL-10 increases seen during exercise are mediated by IL-6 from contracting muscles. Pedersen et al (2003) evaluated young healthy volunteers that received a low dose of recombinant human (rh)IL-6 or saline for $3 \mathrm{~h}$ in the femoral arteries of both leg (approximately $140 \mathrm{pg} / \mathrm{ml}$ ) and observed that musclederived IL-6 plays a key role in exercise-induced enhanced the plasma levels of cortisol, IL-1ra and IL-10 compared with saline infusion. In addition, rhIL-6 induced increased levels in circulating neutrophils and a decrease in the lymphocyte number without effects on plasma epinephrine, body temperature, mean arterial pressure, or heart rate.Cytokines have a short half-life in the circulation. Regardless, levels of IL-10 tend to peak at the cessation of the exercise or shortly thereafter. One previous study shows that after acute exercise $(5-\mathrm{km}$ continuous run on a treadmill at $70 \%$ of speed associated to $\mathrm{VO}_{2} \max$ ) peak concentrations of IL-10 were reached $60 \mathrm{~min}$ after the cessation of acute exercise compared to baseline levels (Cabral-Santos et al. 2016). Thus, the systemic effects induced by IL-10 are for the most part perceived to occur during recovery time from exercise. However, in the present study only IL-10 levels at baseline and immediately after exercise were evaluated due these timepoints that all eligible studies had in common. 


\section{Effects of aerobic exercise}

When observed in cycle ergometer type, Cullen et al (2016) studied the behavior of IL-10 with different intensities and volume in cycle ergometer protocols (Low: $35 \mathrm{~min}$ at 50\% $\mathrm{VO}_{2} \mathrm{max}$; Moderate: 5 bouts of $2 \mathrm{~min}$ at $80 \% \mathrm{VO}_{2} \max$; and High: 5 bouts of $4 \mathrm{~min}$ at $80 \% \mathrm{VO}_{2} \max$ ). However, none of this appears to be sufficient stimulus to induce alterations in plasma or at level of gene expression in peripheral blood of IL-10, independent of being performed continuously or intermittently.

Clearly, physical exercise can be a significant stress to organism and indicators of injury or energy imbalance increase the synthesis and subsequent release of IL-6 from contracting muscle. This cross-talk between skeletal muscle and immune cells may facilitate a broad IL-10 response effects on muscle as well as on different tissue (Pedersen, 2017). Thus, the magnitude of the increase in IL-10 is related to the active muscle mass and therefore exercise intensity.

It has been demonstrated that IL-6 mRNA expression and protein release increase when intramuscular glycogen is low, indicating that IL-6 is a regulator of energy metabolism during exercise (Chan et al. 2004). For the two eligible studies of treadmill running, Ghafourian et al (2016) found no significant differences in plasma IL-10 and IL-10/TNF- $\alpha$ ratio concentrations after 30 minutes of running at $65 \%$ of sVO $\mathrm{s}_{2}$ max, but interleukin- $1 \beta$ (key pro-inflammatory mediator of $\beta$-cell damage in type 2 diabetes mellitus) and soluble intercellular adhesion molecule-1 decrease (sICAM-1) significantly 24 hours after. Once the acute bout of exercise triggers the antiinflammatory effect at least in part mediated by muscle-derived IL-6 release, the current protocol wasn't of high enough intensity to increase the IL-6 release into the circulation and therefore isn't surprising that there was no counter-effect of IL-10 levels.

However, Cabral-Santos et al (2015) found a significant increase in the expression of IL-10 immediately after exercise from baseline (2-fold) after protocol at 70\% $\mathrm{VO}_{2} \mathrm{max}$ - but peak levels were reached 60 min after exercise (4-fold) as well as the IL-10/TNF- $\alpha$ ratio peak. The increase in systemic production of IL-10 carry on augmenting resistance to infections in the immune system function via leukocyte recruitment, enhance B cell survive and differentiation, as well as inhibiting dendritic cells-mediated antigen presenting function limiting immunopathology (Mocellin et al. 2004; Hedrich and Bream, 2010).

In contrast, intermittent protocols may stimulate greater changes in metabolic demand. We identified a total of three articles that verified the relationship between IL-10 and intermittent exercise with the duration of approximately $30 \mathrm{~min}$. Cabral-Santos et al (2015) observed an increase circulating IL-6 values with a peak immediately after exercise, and increased IL-10 levels (208\%) and IL-10/TNF- $\alpha$ ratio with peak at 60 min postexercise in intermittent running protocol (1:1 min at $\mathrm{sVO}_{2} \max$ with passive rest). Similarly, Dorneles et al (2016) found an increase in IL-10 immediately after intermittent exercise (10 x 60s at 85-90\% of Maximal Power OutputPMax with $75 \mathrm{~s}$ active rest at $50 \% \mathrm{PMax}$ ) and peak values at $30 \mathrm{~min}$ post-exercise compared to baseline level, suggesting an anti-inflammatory role promoted by this exercise type. The authors attributed this result to possible large physiological stress, principally due anaerobic metabolism (seen by increase in adrenergic response, cortisol and lactate levels) and changes in markers of muscle damage (observed by increase in lactate dehydrogenase levels) that were positively correlated with leukocyte mobilization into peripheral blood described (including lymphocytes, monocytes and neutrophils).

These results differ from the moderate intermittent protocol in which Dorneles et al (2016) did not find any differences in IL-1ra, IL-8 and IL-10 concentrations $(10 \mathrm{x} 60 \mathrm{~s}$ at $70-75 \%$ with 60 s active intervals at 
50\%PMax). Additionally, Ghafourian et al (2016) observed no significant differences in IL-10 levels after an intermittent protocol comprising 6 × 3min intervals at $85 \%$ of VO2max with a 90 s passive rest, but increased IL$6(139 \%)$ and ratio of IL-6/IL-10 levels (82\%). Thus, the immune modulation (and therefore anti-inflammatory benefits) come with intermittent exercise performed at higher intensities.

In response to amount of time, prior research has showed that in high-intensity intermittent exercises of different exercise volumes (running 1.25 and $2.5 \mathrm{~km}$ with $1: 1 \mathrm{~min}$ at speed associated with VO2max and passive recovery) the increase in IL-10 concentration was related to the duration of exercise indicating the importance of this variable (Cabral-Santos et al. 2016). Taken together, these results showed that changes in IL-10 production is remarkably related to the duration of exercise.

\section{Effects of strength (resistance) exercise}

The effect of strength/resistance exercise on plasma IL-10 is less evident. The dynamics of this type of exercise is influenced by different variables such as intensity, workload, number of repetitions, interval between sets and size of muscle mass involved in muscle contraction. The metabolic cost of this type of exercise is lower compared with aerobic exercise and consequently the IL-10 levels is less pronounced. Agostine et al (2016) utilized strength exercises with 70\% 1RM and observed an increased levels of IL-6 and IL-10 immediately after the exercise which remains elevated post- $1 \mathrm{~h}$ than basal levels. The others studies analyzed did not show this dynamic, although it was observed increase of IL-10 in the period of exercise recovery

Peake et al (2016) tested submaximal (10 sets x 60 at $10 \%$ maximum isometric strength with 1 min rest) versus maximal (10 sets $\times 3$ at 100\% maximum isometric strength with 3 min rest) lengthening contractions performed on an isokinetic dynamometer by elbow flexors. The authors found that the muscle damage (evidenced by creatine kinase activity) was not accompanied by differences in markers of inflammation such as IL-1 ra, IL10 , TNF- $\alpha$ and plasma C-reactive protein (CRP). Since contracting skeletal muscle per se is an important inducer of IL-6, the use of isolated muscle contractions have low metabolic cost compared to aerobic exercises and the increase of circulating cytokine concentrations is less marked (Fisher, 2006), however the absence of systemic changes does not mean that there is no local inflammation response in the exercised muscles.

It is well know that glycogen depletion during contraction leads to the transfer of p38MAPK to the nucleus, which can upregulate IL-6 (Chan et al. 2004), however only higher metabolic demand during the exercise session could influenced the IL-10 response. Rossi et al (2016) performed two randomized sequences at $70 \%$ of 1RM (Short: 30s of rest between sets; Moderate: 90s of rest) with 4 sets until movement failure in the squat and bench press exercises. The authors observed that short rest intervals showed statistically significant decreases in IL-6 and glucose at post-15 of recovery and there were no differences between IL-10 levels for both conditions. This can be attributed, in part, to low exercise volume in short protocol (significantly less weight was lifted and less repetitions executed) adopted by the authors which did not generate sufficient stimulus to increase IL-6.

Interestingly, Gerosa-Neto et al (2016) tested the same protocol but with high intensity (90\% 1RM) and showed that moderate rest interval allowed a higher workload in terms of volume of repetitions and subsequently an increase IL-10 levels (1.9-fold) and IL-10/TNF-a ratio (2.3-fold) compared with the short rest interval condition, but only $30 \mathrm{~min}$ after the cessation of exercise. The differences in volume protocols during the strength exercise session may explained the discrepancy in results between protocols in Rossi et al (2016) and GerosaNeto et al (2016) which were not equalized. 


\section{Effects of duration}

Our results indicate there is a linear relationship between exercise duration and changes in plasma IL-10 (Figure 3). The average IL-10 levels in the strenuous group showed significantly increased up to 5-fold whereas less dramatic increase were more frequent in the other protocols. Thus, the duration of the exercise is the single most important factor determining the magnitude of the exercise-induced increase of plasma IL-10 (48\%).

Krzemiński et al (2016) showed that well-trained young men who completed a $100 \mathrm{~km}$ ultra-endurance exercise reported significant increases in plasma IL-6, IL-10, IL-18 and TNF- $\alpha$ concentrations immediately after the event. At $90 \mathrm{~min}$ of the recovery period, plasma concentrations of IL- 6 and IL-10 were still higher $(\mathrm{p}<0.05)$ than before exercise, whereas plasma TNF- $\alpha$ did not differ significantly from the resting pre-exercise values.

The IL-10 response in this study follows that previously reported for Nickel et al (2012) which showed significantly upregulated levels in all groups compared to their respective baseline levels (10 to 20-fold) after marathon running and that IL-10 values return to baseline values $24 \mathrm{~h}$ after that. It could be argued that this occurrence could prevent potential inflammation and tissue damage. There was an increase in circulating IL-6 secreted by T-cells and macrophages immediately after exercise (7-fold) and remains elevated until 24h after marathon compared to baseline, whereas the increase in TNF- $\alpha$ and C-Reactive Protein was delayed for 24h (7fold).

Kaoru et al (2013) assessed triathletes after a duathlon race comprising $5 \mathrm{~km}$ of running, $40 \mathrm{~km}$ of cycling, and $5 \mathrm{~km}$ of running. There were significant increases in plasma concentrations of IL-6 (30-fold) and IL10 (50-fold) immediately after the race whereas TNF- $\alpha$ did not change. The authors also reported subsequent acute inflammatory responses by increased in IL-8 and monocyte chemoattractant protein-1 (MCP-1); both cytokines are potent neutrophil and monocyte chemokines and stimulate cell extravasation into inflammatory tissue immediately after the race. This may mediate exercise-induced pathogenesis as muscle inflammatory damage, exertional rhabdomyolysis and heat-related multiple organ failure (Kaoru et al. 2013).

Comassi et al (2015) compared the effect between Ironman $(3.8 \mathrm{~km}$ swim, $180 \mathrm{~km}$ cycling and $42.197 \mathrm{~km}$ running without interruption) and Half Ironman competitions on the inflammatory profile in male triathletes. The authors also reported an increase in plasma IL-6 and IL-10 levels (450\% and 146\% respectively) whereas plasma TNF- $\alpha$ did not differ significantly from resting pre-exercise values in both groups. In addition, the authors reported increases in MCP-1 levels and increased white blood cells count, attributed to cell mobilization in response to the increase in mechanical and metabolic stress observed in both groups. This study reported a marked increase in IL-6 and IL-10 levels provoked by exercise and direct anti-inflammatory effects by limiting of TNF- $\alpha$ and IL-1 $\beta$ signaling.

However, studies indicate the pronounced inflammatory response induced by prolonged and exhaustive exercise could lead to transient suppression of several immune components and increase the risk of upper respiratory symptoms, potentially due to the cross-regulatory effect of interleukin- 4 on interferon- $\gamma$ production and immunosuppressive action of IL-10 (Gleeson, 2007; Shaw et al. 2018). To prevent transient suppression of the immune system and in illness prone athletes, those negaged in strenuous training programs might need to adopt effective strategies to support the immune function.

Overall completeness and applicability of evidence/Implications for practice 
Physical activity represents a natural anti-inflammatory effects. Due the methodological heterogeneity in the protocols identified, our review shows that manipulations in duration of the exercise exerts direct inflammatory effects in magnitude on release IL-10.

Despite the duration of exercise appears be the single most important factor, currently data lead to hypothesize that additional influence of intensity could determine this magnitude. Although the intensity was not significantly associated with circulating levels of IL-10, there was a linear relationship.

Risk of bias

Some potential limitations could affect the interpretation of our findings. First, the sample size in these studies was small, so the distribution of the data was not normal. Second, the analysis was based on unadjusted data and did not control for confounding factors including gender, age, previous physical conditioning, and therefore cannot exclude the influence of mixed factors.

\section{Authors' contributions}

RZAP, JSRN, NCB and FSL designed the study protocol. CCS, IMCF and EALJ conducted the search and screening of titles, abstracts, full-text articles, the study selection, data extraction and quality, screened the fulltext articles, and assessed the eligibility of the studies. CCS and EALJ conducted the analysis and interpretation of data and contributed to the writing of the manuscript. All authors read the final version of the manuscript.

\section{Conflict of Interest}

The authors declare that they have no conflict of interest. 


\section{References}

Abbasi, A. , Hauth, M. , Walter, M. , Hudemann, J. , Wank, V., Niess, A. M. , \& Northoff, H. (2014) Exhaustive exercise modifies different gene expression profiles and pathways in LPS-stimulated and un-stimulated whole blood cultures. Brain, Behavior, and Immunity, 39, 130-141. https://doi.org/10.1016/j.bbi.2013.10.023

Agostinete, R. R., Rossi, F. E. , Magalhaes, A. J. , Rocha, A. P., Parmezzani, S. S. , Gerosa-Neto, J., Cholewa, J. M. , \& Lira, F. S. (2016) Immunometabolic Responses after Short and Moderate Rest Intervals to Strength Exercise with and without Similar Total Volume. Frontiers in Physiology, 7, 444. https://doi.org/10.3389/fphys.2016.00444

Beutler, B. A. (1999) The role of tumor necrosis factor in health and disease. Journal of Rheumatology Supplement, $57,16-21$

Cabral-Santos, C. , Gerosa-Neto, J. , Inoue, D. S. , Panissa, V. L. G. , Gobbo, L. A. , Zagatto, A. M. , Campos, E. Z. , \& Lira F. S. (2015) Similar Anti-Inflammatory Acute Responses from Moderate-Intensity Continuous and High-Intensity Intermittent Exercise. Journal of Sports Science and Medicine, 14, 849-856.

Cabral-Santos, C. , Castrillon, C. I. M. , Miranda, R. A. T. , Monteiro, P. A. , Inoue, D. S. , Campos, E. Z. , Hofmann, P. , \& Lira, F. S. (2016) Inflammatory Cytokines and BDNF Response to High-Intensity Intermittent Exercise: Effect the Exercise Volume. Frontiers in Physiology, 7, 509. https://doi.org/10.3389/fphys.2016.00509

Chan, M. H. , McGee, S. L. , Watt, M. J. , Hargreaves, M. , \& Febbraio, M. A. (2004) Altering dietary nutrient intake that reduces glycogen content leads to phosphorylation of nuclear p38 MAP kinase in human skeletal muscle: association with IL-6 gene transcription during contraction. FASEB Journal, 18, 1785-1787. https://doi.org/10.1096/fj.03-1039fje

Comassi, M. , Vitolo, E. , Pratali, L. , Del Turco, S. , Dellanoce, C. , Rossi, C. , Santini, E. , \& Solini, A. (2015) Acute effects of different degrees of ultra-endurance exercise on systemic inflammatory responses. Journal of Internal Medicine, 45, 74-79. https://doi.org/10.1111/imj.12625

Cullen, T., Thomas, A. W. , Webb, R. , \& Hughes, M. G. (2016) Interleukin-6 and associated cytokine responses to an acute bout of high-intensity interval exercise: the effect of exercise intensity and volume. Applied Physiology, Nutrition, and Metabolism, 41, 803-808. https://doi.org/10.1139/apnm-2015-0640

de Waal Malefyt, R. , Abrams, J. , Bennett, B. , Figdor, C. G. , \& de Vries, J. E. (1991) Interleukin 10 Inhibits Cytokine Synthesis Human Monocytes: An Autoregulatory Role of IL-10 Produced by Monocytes. Journal of Experimental Medicine, 174, 1209-1220. https://doi.org/10.1084/jem.174.5.1209 
Dorneles, G. P. , Haddad, D. O. , Fagundes, V. O., Vargas, B. K. , Kloecker, A., Romão, P. R. , \& Peres, A. (2016) High intensity interval exercise decreases IL-8 and enhances the immunomodulatory cytokine interleukin10 in lean and overweight-obese individuals. Cytokine, 77, 1-9. https://doi.org/10.1016/j.cyto.2015.10.003

Fiorentino, D.F., Bond, M. W. , \& Mosmann, T. R. (1989) Two types of mouse T helper cell. IV. Th2 clones secrete a factor that inhibits cytokine production by Th1 clones. Journal of Experimental Medicine, 170, 20812095. https://doi.org/10.1084/jem.170.6.2081

Fischer, C. P. (2006) Interleukin-6 in acute exercise and training: what is the biological relevance? Exercise Immunology Review, 12, 6-33.

Gerosa-Neto, J. , Rossi, F. E. , Campos, E. Z. , Antunes, B. M. , Cholewa, J. M. , \& Lira, F. S. (2016) Impact of Short and Moderate Rest Intervals on the Acute Immunometabolic Response to Exhaustive Strength Exercise: Part II. Journal of Strength and Conditioning Research, 30, 1570-1576.

Ghafourian, M. , Ashtary-Larky, D. , Chinipardaz, R. , Eskandary, N. , \& Mehavaran, M. (2016) Inflammatory Biomarker Response to Two Different Intensities of a Single Bout Exercise Among Soccer Players. Iranian Red Crescent Medical Journal, 18, e21498.

Gleeson, M. (2007) Immune function in sport and exercise. Journal of Applied Physiology, 103, 693-699. https://doi.org/10.1152/japplphysiol.00008.2007

Hedrich, C. M. , \& Bream, J. H. (2010) Cell type-specific regulation of IL-10 expression in inflammation and disease. Journal of Immunology Research, 47, 185-206. https://doi.org/10.1007/s12026-009-8150-5

Iyer, S. S. , \& Cheng, G. (2012) Role of Interleukin 10 Transcriptional Regulation in Inflammation and Autoimmune Disease. Critical Reviews in Immunology, 32, 23-63.

Kaoru, S. , Suzuki, K. , Yoshitani, K. , Shiraishi, K. , \& Kometani, T. (2013) Urinary excretion of cytokines versus their plasma levels after endurance exercise. Exercise Immunology Review, 19, 29-48.

Krzemiński, K. , Buraczewska, M. , Miśkiewicz, Z. , Dąbrowski, J. , Steczkowska, M. , Kozacz, A. , \& Ziemba, A. (2016) Effect of ultra-endurance exercise on left ventricular performance and plasma cytokines in healthy trained men. Biology of Sport, 33, 63-69. http://doi.org/10.5604/20831862.1189767 
Kwilasz, A. J., Grace, P. M. , Serbedzija, P., Maier, S. F. , \& Watkins, L. R. (2015) The therapeutic potential of interleukin-10 in neuroimmune diseases. Neuropharmacology, 96, 55-69. https://doi.org/10.1016/j.neuropharm.2014.10.020

Lira, F. S. , Yamashita, A. S. , Rosa, J. C. , Koyama, C. H. , Caperuto, E. C. , Batista, M. L. Jr. , \& Seelaender, M. C. (2012) Exercise training decreases adipose tissue inflammation in cachectic rats. Hormone and Metabolic Research, 44, 91-98.

Mocellin, S. , Marincola, F. , Rossi, C. R. , Nitti, D. , \& Lise, M. (2004) The multifaceted relationship between IL10 and adaptive immunity: putting together the pieces of a puzzle. Cytokine \& Growth Factor Reviews, 15, 61-76. https://doi.org/10.1016/j.cytogfr.2003.11.001

Moseley, A. M. , Herbert, R. D., Sherrington, C. , \& Maher, C. G. (2002). Evidence for physiotherapy practice: A survey of the physiotherapy evidence database (PEDro). Australian Journal of Physiotherapy, 48, 43-49. https://doi.org/10.1016/S0004-9514(14)60281-6

Neubauer, O. , König, D. , \& Wagner, K. H. (2008) Recovery after an Ironman triathlon: sustained inflammatory responses and muscular stress. European Journal of Applied Physiology, 104, 417-426. https://doi.org/10.1007/s00421-008-0787-6

Nickel, T. , Emslander, I. , Sisic, Z. , David, R., Schmaderer, C. , Marx, N. , Schmidt-Trucksäss, A. , Hoster, E. , Halle, M. , Weis, M. , \& Hanssen, H. (2012) Modulation of dendritic cells and toll-like receptors by marathon running. European Journal of Applied Physiology, 112, 1699-1708. https://doi.org/10.1007/s00421-011-2140-8

Oswald, I. P. , Wynn, T. A., Sher, A. , \& James, S. L. (1992) Interleukin 10 inhibits macrophage microbicidal activity by blocking the endogenous production of tumor necrosis factor alpha required as a costimulatory factor for interferon gamma-induced activation. Proceedings of the National Academy of Sciences, 89, 8676-80. https://doi.org/10.1073/pnas.89.18.8676

Peake, J. M. , Nosaka, K. , Muthalib, M. , \& Suzuki, K. (2006) Systemic inflammatory responses to maximal versus submaximal lengthening contractions of the elbow flexors. International Society of Exercise and Immunology, 12, 72-85.

Pedersen, B. K. , \& Febbraio, M. A. (2009) Muscle as an endocrine organ: focus on muscle-derived interleukin-6. Physiological Reviews, 88, 1379-1406. https://doi.org/10.1152/physrev.90100.2007 
Pedersen, B. K. , \& Saltin, B. (2015). Exercise as medicine - evidence for prescribing exercise as therapy in 26 different chronic diseases. Scandinavian Journal of Medicine \& Science in Sports, 3, 1-72. https://doi.org/10.1111/sms.12581

Pedersen, B. K. (2017) Anti-inflammatory effects of exercise: role in diabetes and cardiovascular disease. European Journal of Clinical Investigation, 47, 600-611. https://doi.org/10.1111/eci.12781

Rosa-Neto, J. C. , Lira, F. S. , Oyama, L. M. , Zanchi, N. E. , Yamashita, A. S. , Batista, M. L. Jr. , Oller do Nascimento, C. M. , \& Seelaender, M. (2009) Exhaustive exercise causes an anti-inflammatory effect in skeletal muscle and a pro-inflammatory effect in adipose tissue in rats. European Journal of Applied Physiology, 106, 697704. https://doi.org/10.1007/s00421-009-1070-1

Rossi, F. E. , Gerosa-Neto, J. , Zanchi, N. E. , Cholewa, J. M. , \& Lira, F. S. (2016) Impact of Short and Moderate Rest Intervals on the Acute Immunometabolic Response to Exhaustive Strength Exercise: Part I. Journal of Strength and Conditioning Research, 30, 1563156-9.

Shaw, D. M. , Merien, F. , Braakhuis, A. , \& Dulson, D. (2018) T-cells and their cytokine production: The antiinflammatory and immunosuppressive effects of strenuous exercise. Cytokine, 104, 136-142. https://doi.org/10.1016/j.cyto.2017.10.001

Shek, P. N. , \& Shephard, R. J. (1998) Physical exercise as a human model of limited inflammatory response. Canadian Journal of Physiology and Pharmacology, 76, 589-597.

Steensberg, A. , Fischer, C. P. , Keller, C. , Møller, K. , \& Pedersen, B. K. (2003) IL-6 enhances plasma IL-1ra, IL-10, and cortisol in humans. American Journal Of Physiology-Endocrinology And Metabolism, 285, E433-E437.

Wadley, A. J. , Chen, Y. W. , Lip, G. Y. , Fisher, J. P. , \& Aldred, S. (2016). Low volume-high intensity interval exercise elicits antioxidant and anti-inflammatory effects in humans. Journal of Sports Sciences, 34, 1-9. https://doi.org/10.1080/02640414.2015.1035666

Wang, P. , Wu, P. , Siegel, M. I. , Egan, R. W. , \& Billah, M. M. (1994) IL-10 inhibits transcription of cytokine genes in human peripheral blood mononuclear cells. Journal of Immunology, 153, 811-816. 


\section{Figures List}

Figure 1. Inclusion flowchart of the selected studies.

Figure 2. Effect of different types of acute exercise and the corresponding increase in plasma IL-10 (delta change from pre-exercise level) in humans.

Based on the 12 studies, the graphs represent approximately 164 subjects (listed in Table 1). Each dot represents one exercise protocol, while the corresponding bars show geometric means and standard error of mean.

Figure 3. Linear relation between exercise duration and changes in plasma IL-10.

Each dot represents one exercise protocol.

Figure 4. Linear relation between exercise intensity and changes in plasma IL-10.

Each dot represents one exercise protocol. 
Table List

Table 1. Characteristics of the included studies.

Legend $=$ Values are mean \pm standard deviation. Abbreviations: $1 \mathrm{RM}=$ One maximum repetition, $\mathrm{MaxHR}=$ maximal heart rate, PMax $=$ Maximal potency; $\uparrow=$ increase; $\downarrow=$ decrease; $\leftrightarrow=$ no diferences in IL-10 levels. In studies investigating the effect of any intervention, only the result from the control group exercise is presented. 


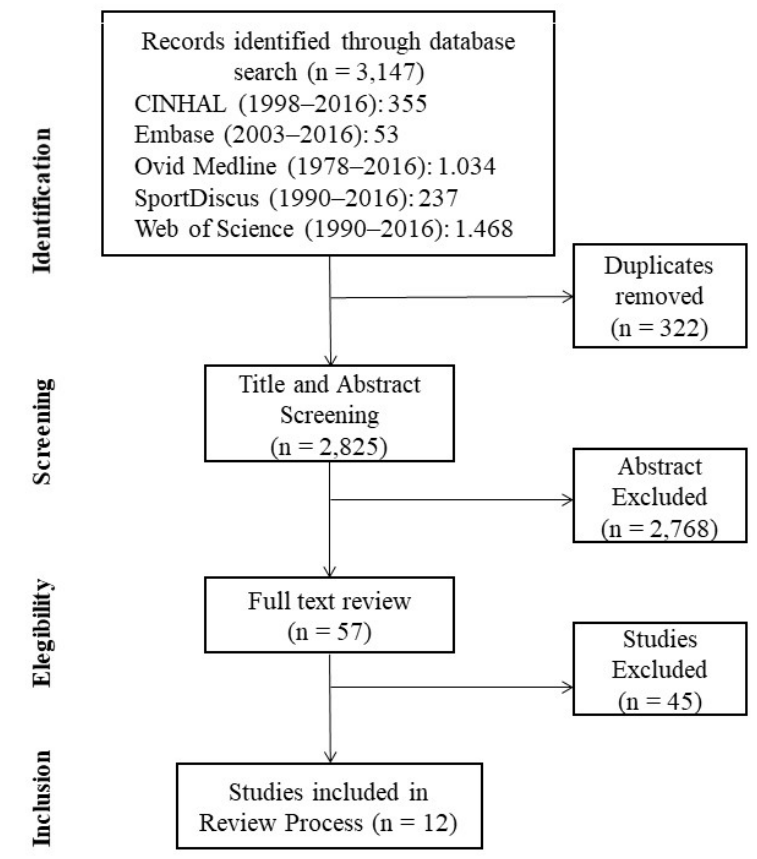

Figure 1. Inclusion flowchart of the selected studies. 


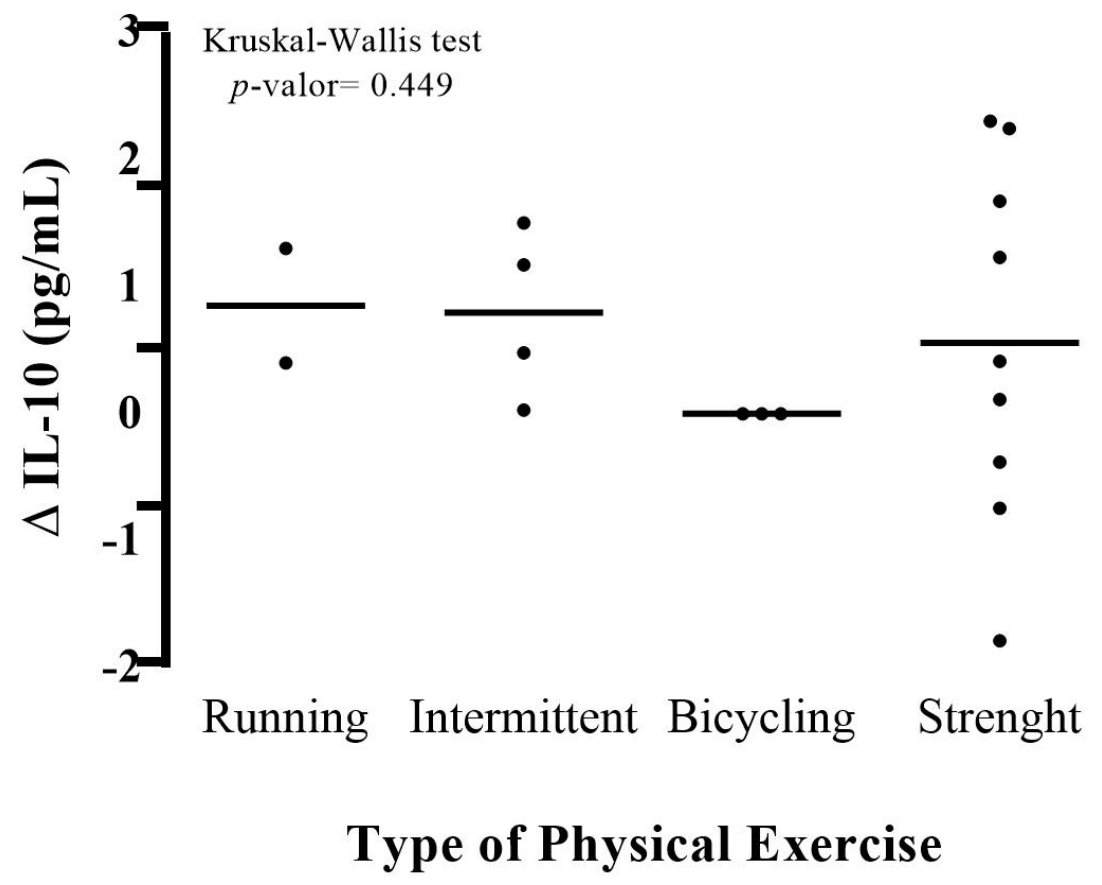

Figure 2. Effect of mode of acute exercise on plasma IL-10 in humans.

Legend $=$ In studies investigating the effects of a intervention on the IL-10 response to acute exercise is presented. 


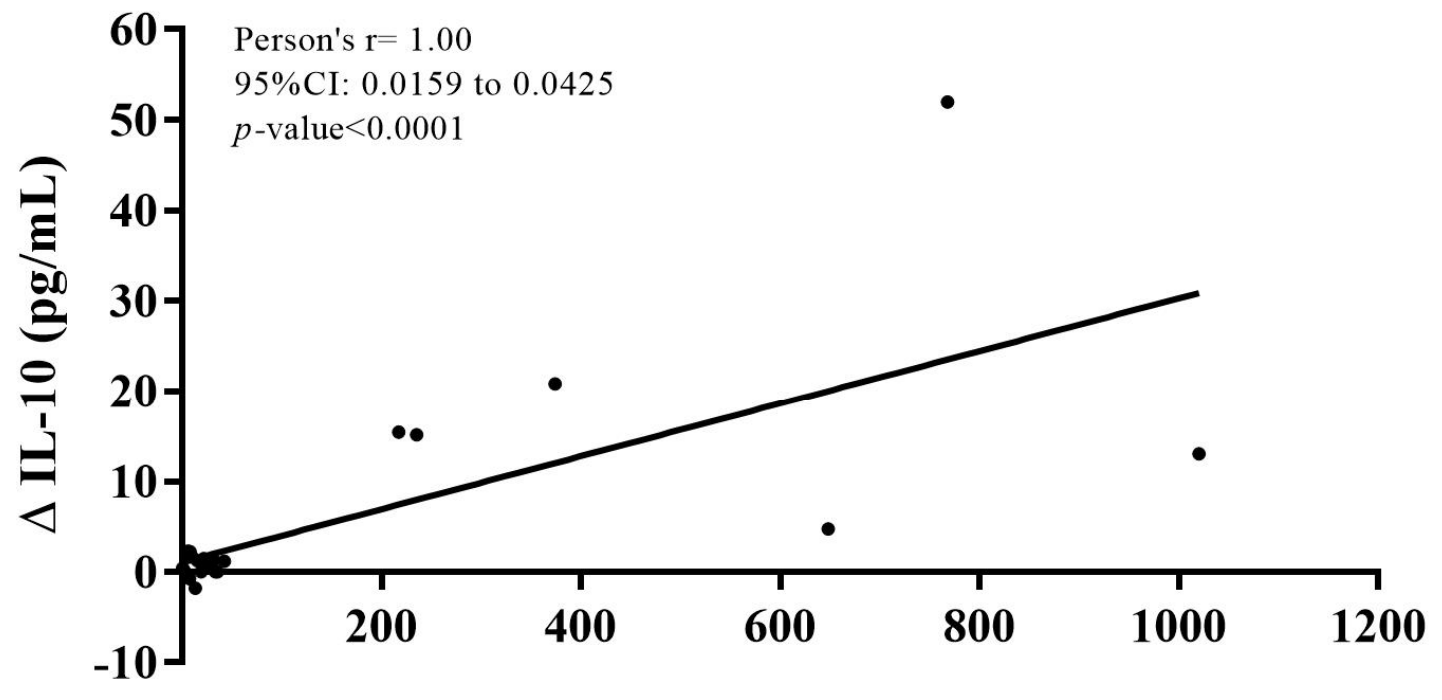

Exercise Duration (min)

Figure 3. Linear relation between exercise duration and changes in plasma IL-10. 


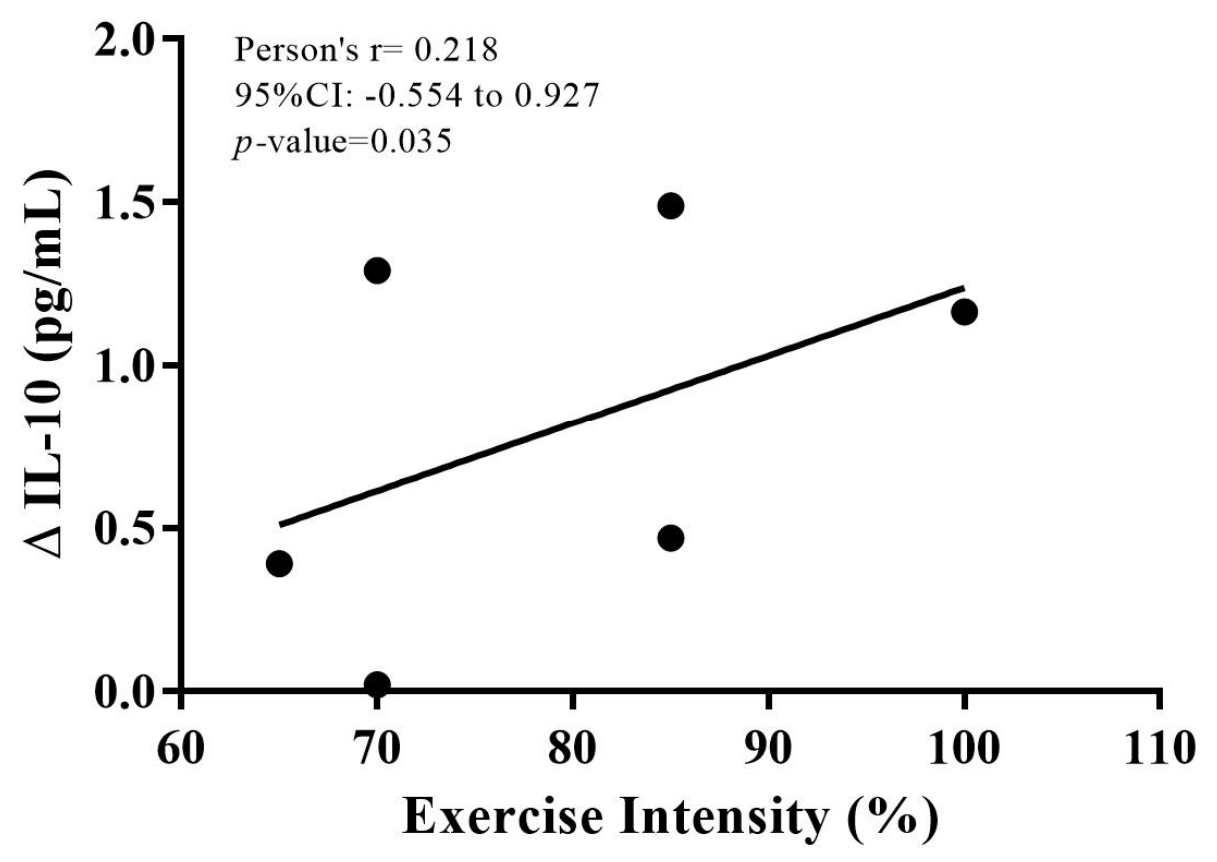

Figure 4. Linear relation between exercise intensity and changes in plasma IL-10. 
TAB LE I Characteristics of the included studies

$\begin{array}{ccl}\text { Reference } & \text { Characteristics of participants } & \text { Design } \\ \text { Agostinete et al. } & \text { Eight physically active healthy men, } & \text { Three randomized session: } \\ \text { (2016) (Brazil) } & 23.5 \pm 3.1 \text { years, } 80.7 \pm 6.4 \mathrm{~kg} & \text { Short: } 70 \% 1 \mathrm{RM}, 30 \mathrm{~s} \text { of rest; } \\ & & \text { Moderate: } 70 \% 1 \mathrm{RM}, 90 \mathrm{~s} \text { of rest; } \\ & \begin{array}{l}\text { Volume-equated Short: } 70 \% 1 \mathrm{RM}, 30 \mathrm{~s} \\ \text { of rest }\end{array}\end{array}$
ergometer:

Cullen et al. (2016) 10 healthy active individuals (ive years, $1.70 \pm 0.9 \mathrm{~m}, 67 \pm 11 \mathrm{~kg}$ $\mathrm{VO}_{2} \max 49 \pm 5 \mathrm{ml} / \mathrm{kg} / \mathrm{min}$

Low: 35 min cycling at $50 \% \mathrm{VO}_{2} \max$;

Moderate: $5 \times 5 \mathrm{~min}$ bouts at $50 \% \mathrm{VO}_{2} \max$

interspersed 2 min intervals at $80 \%$

$\mathrm{VO}_{2}$ max;

High: $5 \times 4$ min bouts at $80 \% \mathrm{VO}_{2} \max$

interspersed with $3 \mathrm{~min}$ intervals at $50 \%$

$\mathrm{VO}_{2} \max$

High-intensity exercise (HIIE): 10 bouts $x$

$60 \mathrm{~s}(85-90 \% \mathrm{PMax})$ interspersed $75 \mathrm{~s}$

active (50\%PMax); and

(2016) (Brazi)

individuals $(n=10)$ with

$26.5 \pm 6.11$ years

Moderate exercise (MIIE): 10 bouts $\times 60$ s (70-75\% PMax) interspersed $60 \mathrm{~s}$ active (50\%PMax)

The duration of exercise was approximately HIIE
Exercise protocol

Four sets of Squat, Bench Pess and T-

Barrow exercises until movement failure

$30 " \uparrow$

PEDRO escore

Pos: 5.
$90 "$

$90 " \uparrow$

Pre: $2.93 \pm 2.64$

Pos: $4.59 \pm 4.61$

Volume-equated

Pre: $2.94 \pm 5.17$

Pos: $3.06 \pm 4.90$

HIIE: 5 -km run on a treadmill performed in HIIE $\uparrow$ intermittently at $1-\mathrm{min}$ at the $\mathrm{SVO}_{2}$ peak Pre: $1.389 \pm 0.407$ followed by 1 -min of pasive recovery Pos: $2.553 \pm 0.913$ treadmill at SSE $70 \%$ of $\mathrm{sVO}_{2}$ peak Pos: $2.436 \pm 1.508$

The duration of exercise was

$12: 48: 21 \pm 1: 14: 18 \mathrm{hr}$ for IR and $6: 14: 43 \pm 0: 37 \cdot 25 \mathrm{hr}$ for HIR

IR $\uparrow$

Pre: $11.5 \pm 6.6$

Pos: $63.5 \pm 65.9$
HIR $\uparrow$

Pre: $14.2 \pm 19.2$

Pos: $35.0 \pm 32.8$

LOW $\downarrow$

Pre: $0.17 \pm 0.06 \mathrm{pg} / \mathrm{ml}$

MOD $\downarrow$

Pre: $0.18 \pm 0.09 \mathrm{pg} / \mathrm{m}$ Pos: $0.17 \pm 0.07 \mathrm{pg} / \mathrm{m}$ $\mathrm{HIGH} \downarrow$

Pre: $0.13 \pm 0.07 \mathrm{pg} / \mathrm{ml}$

Pos: $0.12 \pm 0.09 \mathrm{pg} / \mathrm{ml}$

Pre: $2.48 \pm 0,58$

Pos: $3.97 \pm 1.62$

MIIE $\leftrightarrow$ Pre: $1.67 \pm 0.74$

Pos: $1.69 \pm 0.85$ 


\section{Reference}

Gerosa-Neto et al. 8 healthy subjects age $25.2 \pm 4.1$

(2016) (Brazil)

years, $76.4 \pm 7.7 \mathrm{~kg}, 1.78 \pm 0.10 \mathrm{~m}$

hafourian et al.

20 healthy man

(2016) (Iran)

$21.3 \pm 1.6$ years, $74.1 \pm 8.3 \mathrm{~kg}$,
$1.80 \pm 0.6 \mathrm{~m}, \mathrm{VO}_{2} \max 53 \pm 4$.

$1.80 \pm 0.6$
$\mathrm{~kg} / \mathrm{min}$

\section{Design}

Two randomized sessions with different

rest intervals on the treadmill:

Short: $90 \%$ of 1 RM and $30 \mathrm{~s}$ rest allowed

between sets;

Moderate: $90 \%$ of $1 \mathrm{RM}$ and $90 \mathrm{~s}$ rest

allowed between sets

Two protocols of a single bout of treadmill:

Submaximal: $30 \mathrm{~min}$ of running at a speed

of $65 \%$ of $\mathrm{VO}_{2} \max$

Maximal: six periodic repetitions with three minutes at a speed of $85 \%$ of $\mathrm{VO}_{2} \max$

with $90 \mathrm{~s}$ of rest among each repetition

Kaoru et al. (2013) 14 male triathletes, $28.7 \pm 7.9$ years, One session of duathlon race

(Japan)

$63.2 \pm 6.0 \mathrm{~kg}$

Krzemiński et al.

(2016) (Poland)

9 healthy trained male $30.0 \pm 1.0$

A 100-km ultra-marathon (runners had to years, $720 \pm 2.0 \mathrm{~kg}, 1.78 \pm 0.20 \mathrm{~m}$ $\mathrm{VO}_{2} \max 54.1 \pm 3.0 \mathrm{~m} / \mathrm{kg} / \mathrm{min}$

Nickel et al. (2011) 16 lean elite group (regular intensive exercise $>55 \mathrm{~km} /$ week): $74.4 \pm 11 \mathrm{~kg} ; 40 \pm 7$ years, BMI $22 \pm 1 \mathrm{kgm}^{2}$ exercise $<40 \mathrm{~km} /$ week):

$78.5 \pm 9 \mathrm{~kg}, 40 \pm 6$ years, BM

$24 \pm 2 \mathrm{kgm}^{2}$;

Peake et al. (2006) 10 healthy young men, $22.9 \pm 4.7$ (Japan) years, $76.2 \pm 11.8 \mathrm{~kg}, 1.80 \pm 0.08 \mathrm{~m}$

Cross-over design Submaximal: 10 sets of 60 lengthening contractions at $10 \%$

maximum isometric strength, or Maximal:

10 sets of three lengthening contraction

at $100 \%$ maximum isometric strength

Rossi et al. (2016) Eight healthy subjects with strength Two randomized sessions with different (Brazil) training experience, $24.6 \pm 4.1$ years, $76.4 \pm 7.7 \mathrm{~kg}$
16 lean nonelite (regularintensive rest intervals:

Short: $70 \%$ of $1 \mathrm{RM}$ with $30 \mathrm{~s}$ of rest

between sets, or

Moderate: $70 \%$ of $1 \mathrm{RM}$ with $90 \mathrm{~s}$ of rest between sets
Exercise protocol

Four sets until movement failure in the

squat and bench press exercises
Outcome IL-10 (pg/ml)

90" $\leftrightarrow$ Pre: $18.2 \pm 12.7$

Pos: $16.4 \pm 10.7$

$30 " \leftrightarrow$

Pre: $17.0 \pm 10.0$

Pos: $16.6 \pm 10.9$

$65 \% \mathrm{VO}_{2} \max \leftrightarrow$ Pre: $2.19 \pm 0.20$

Pos: $2.58 \pm 0.45$

$85 \% \mathrm{VO}_{2} \max \uparrow$

Pre: $2.91 \pm 0.77$

Pos: $3.38 \pm 0.37$

The race consisted of $5 \mathrm{~km}$ of running

$40 \mathrm{~km}$ of cycling, and $5 \mathrm{~km}$ of

Pre: $3.1 \pm 8.2 \uparrow$

Pos: $16.1 \pm 31.4$

running ( 17 hr)

Measurements at pre, $0,1.5$, and $3 \mathrm{hr}$

after race

The runners were allowed to choose their own speed and had free access to fluids and carbohydrate-rich food available in support tents at the refreshment points Race results

LE: $217 \pm 28 \mathrm{~min}$

LNE: $235 \pm 28 \mathrm{~min}$

Pre: 1.48

Pos: 16.9

Pre: $0.31 \pm 0.06$

Pos: $5.04+1.34$

LNE $\uparrow$

Pre: 1.47

Pos: 16.6

Elbow flexor on preacher curl bench placed alongside an isokinetic dynamometer

Submaximal $\leftrightarrow$ Pre: $1.7 \pm 1.3$ Pos: $2.1 \pm 1.9$

Maximal $\leftrightarrow$ Pre: $1.5 \pm 1.4$

after, $3 \mathrm{hr}, 1,2,3$ and 4 days after

Pos: $1.6 \pm 1.8$

Four sets of squat and bench press until movement failure

\section{Short $\leftrightarrow$ Pre: $16.30 \pm 9.88$}

Pos: $17.52 \pm 12.83$

Moderate $\leftrightarrow$ Pre: $16.64 \pm 11.86$

Pos: $15.88 \pm 11.3$
PEDRO escore

Note. Values are the mean \pm standard deviation. In studies investigating the effect of any intervention, only the result from the control group exercise is presented. 1RM: One maximum repetition; MaxHR: maximal heart rate; PMa: maximal potency; $\uparrow$ : increase; $\downarrow$ : decrease; $\leftrightarrow$ : no diferences in IL-10 levels. 


\section{Appendix 1}

\section{Search Algorithm}

- Outcome

1. inflamm\$ OR

2. biomarkers OR

3. immune\$ OR

4. inflammatory mediators OR

5. inflammatory biomarkers OR

6. serum mediators OR

7. interleukin-10 OR

8. IL-10 AND

- Population

9. health $\$$ OR

10. healthy individuals AND

- Intervention

11. physical exercise [MeSH Terms],

12. physical training $\mathrm{OR}$,

13. continuous exercise OR,

14. high intensity interval exercise OR,

15. high intensity interval training OR,

16. sprint interval training OR,

17. sprint interval exercise OR,

18. resistance exercise OR,

19. endurance exercise OR,

20. aerobic exercise OR,

21. strength exercise. 